\title{
Erratum to: Managing chronic pain: results from an open-label study using MC5-A Calmare ${ }^{\circledR}$ device
}

\author{
Marianna Ricci - Sara Pirotti • Emanuela Scarpi • \\ Marco Burgio - Marco Maltoni • Elisabetta Sansoni • \\ Dino Amadori
}

Published online: 30 December 2012

(C) Springer-Verlag Berlin Heidelberg 2012

Erratum to: Support Care Cancer (2012) 20:405-412

DOI 10.1007/s00520-011-1128-6

Marco Maltoni was incorrectly affiliated to Palliative Care Unit, Forlimpopoli Hospital, Via Duca d'Aosta 33, 47034 Forlimpopoli (FC), Italy. The correct affiliation of Marco Maltoni is Palliative Care Unit, IRCCS Istituto Scientifico Romagnolo per lo Studio e la Cura dei Tumori (IRST), Meldola, Italy.

The online version of the original article can be found at http://dx.doi.org/ 10.1007/s00520-011-1128-6.

M. Ricci $\cdot$ E. Scarpi $\cdot$ M. Burgio $\cdot$ D. Amadori Istituto Scientifico Romagnolo per lo Studio e la Cura dei Tumori (IRST),

Via P. Maroncelli 40,

47014 Meldola, FC, Italy

S. Pirotti $\cdot$ E. Sansoni

Palliative Care Unit, Forlimpopoli Hospital,

Via Duca d'Aosta 33,

47034 Forlimpopoli, FC, Italy

M. Maltoni $(\square)$

Palliative Care Unit, IRCCS Istituto Scientifico Romagnolo per lo

Studio e la Cura dei Tumori (IRST),

Meldola, Italy

e-mail: ma.maltoni@ausl.fo.it 